\title{
On the stability limits of long nonaxisymmetric cylindrical liquid bridges
}

F. Zayas

E.T.S.I. Industriales, Universidad de Extremadura, 06071 Badajoz, Spain

J. I. D. Alexander

Department of Mechanical and Aerospace Engineering and National Center for Microgravity Research on Fluids and Combustion, Case Western Reserve University, Cleveland, Ohio 44106

J. Meseguer

IDR/UPM, E.T.S.I. Aeronáuticos, Universidad Politécnica de Madrid, 28040 Madrid, Spain

\section{J.-F. Ramus}

Department of Mechanical and Aerospace Engineering and National Center for Microgravity Research on Fluids and Combustion, Case Western Reserve University, Cleveland, Ohio 44106

(Received 12 August 1999; accepted 23 December 1999)

There is a self-similar solution for the stability limits of long, almost cylindrical liquid bridges between equal disks subjected to both axial and lateral accelerations. The stability limits depend on only two variables; the so-called reduced axial, and lateral Bond numbers. A novel experimental setup that involved rotating a horizontal cylindrical liquid bridge about a vertical axis of rotation was designed to test the stability limits predicted by the self-similar solution. Analytical predictions compared well with both numerical and experimental results. (C) 2000 American Institute of Physics. [S1070-6631(00)01704-9]

\section{INTRODUCTION}

Liquid bridges are volumes of liquid held between solids by surface tension forces. They occur in both natural and technological situations and have been studied for practical reasons and for basic scientific interest. In this article we consider the stability of a cylindrical liquid bridge to nonaxial acceleration. The bridge consists of an isothermal drop of liquid held by surface tension forces between two parallel, coaxial, solid disks of the same diameter as shown in Fig. 1. The equilibrium interface shape, $r=F(z, \theta)$, of such a liquid bridge configuration is determined by the following dimensionless parameters: the slenderness, $\Lambda=L /(2 R)$, the axial Bond number, $B_{a}=\Delta \rho a_{a} R^{2} / \sigma$, and the lateral Bond number, $B_{l}=\Delta \rho a_{l} R^{2} / \sigma$, and a dimensionless volume $V$ $=V^{*} /\left(\pi R^{2} L\right)$ defined as the ratio of the actual volume $V^{*}$ to the volume of a cylinder of the same length and diameter. Here $L$ and $R$ are the distance between the disks and the disk radius, respectively. The difference between the density of the liquid and the density of the surrounding medium is $\Delta \rho$, $a_{a}$, and $a_{l}$, are the axial and lateral components of the acceleration acting on the liquid, as indicated in Fig. 1, and $\sigma$ is the surface or interfacial tension. In this article, our analysis is restricted to bridges with volumes close to $V=1$ (cylindrical volumes).

The stability of a liquid bridge with a cylindrical volume depends on the values of the slenderness and the nature of the imposed perturbation. Equilibrium shapes and stability limits of capillary liquid bridges have been investigated theoretically and experimentally for some time, and there is an extensive body of literature dealing with such fluid configurations (see, for example, Ref. 1). However, most of the published articles deal with axisymmetric liquid bridges (in which the direction of the acceleration is parallel to the liquid bridge axis, that is $B_{l}=0$ ), and, apart from the earlier work of Coriell, Hardy, and Cordes, ${ }^{2}$ nonaxisymmetric liquid bridges have been considered only recently. An asymptotic analysis concerning the influence of lateral Bond number on the stability limit of liquid bridges having cylindrical volume $(V=1)$ was published by Perales. ${ }^{3}$ The combined effect of nonaxial acceleration, in the form of a lateral Bond number and noncoaxial supporting disks on the stability limit of cylindrical volume liquid bridges was analyzed both analytically and experimentally in Ref. 4. More recently the equilibrium shapes and stability limits of nonaxisymmetric liquid bridges were examined ${ }^{5-9}$ and nonaxisymmetric configurations appear in an experimental work which explores the use of liquid bridges as accelerometers. ${ }^{10}$

An asymptotic analysis of the stability limits of liquid bridges was conducted by Meseguer et al. ${ }^{4}$ According to their results, for a liquid bridge between equal coaxial disks, close to the cylindrical volume and subjected to both axial and lateral Bond numbers, the maximum stable slenderness, $\Lambda_{\max }$, becomes:

$$
\Lambda_{\max }=\pi\left[1-\left(\frac{3}{2}\right)^{4 / 3} B_{a}^{2 / 3}+\frac{1}{2} v-\frac{\pi^{2}}{4} B_{l}^{2}\right],
$$

where $v=V-1$. This expression can be written in a more compact form by introducing reduced axial and lateral Bond numbers defined as follows:

$$
\begin{aligned}
& b_{a}=\left(\frac{3}{2}\right)^{2} \frac{B_{a}}{\lambda^{3 / 2}}, \\
& b_{l}=\frac{\pi}{2} \frac{B_{l}}{\lambda^{1 / 2}},
\end{aligned}
$$




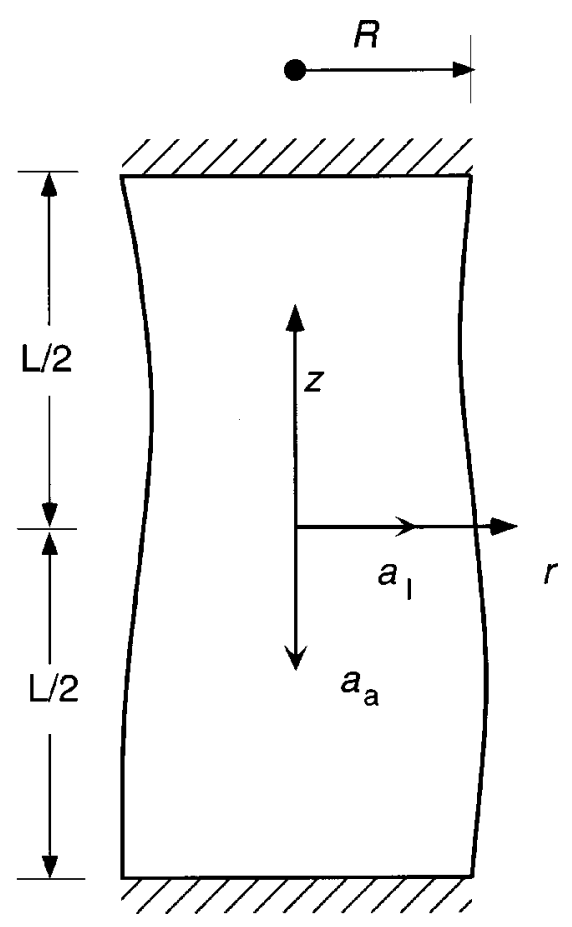

FIG. 1. The liquid bridge set-up.

where $\lambda=1-\Lambda_{\max } / \pi+v / 2$. Substituting the above expressions in Eq. (1) yields

$$
b_{l}=\left(1-b_{a}^{2 / 3}\right)^{1 / 2} \text {. }
$$

This seems to indicate that, at least close to the reference configuration $\left(\Lambda \rightarrow \pi, v \rightarrow 0, B_{a} \rightarrow 0, B_{l} \rightarrow 0\right)$, there is a selfsimilar solution for the stability limits of liquid bridges. That is, the stability limit is independent of the slenderness, $\Lambda$ or volume, $V$.

The range of validity of Eq. (4) was investigated through a set of experiments and through numerical calculations. The stability limits of liquid bridges subjected to both axial and lateral Bond numbers have been obtained and compared to (4). It is implicit in (4) that the Bond numbers are small. Since $B=\Delta \rho a R^{2} / \sigma$, it can be made small by reducing the acceleration, $a$, acting on the liquid column. This can be achieved in a free-fall experiment (for example in a drop tower, on aircraft flying parabolic flight trajectories, in sounding rockets, and on low-earth orbit platforms). The Bond number can also be reduced by matching the densities of the working liquid and the surrounding fluid (the plateau or neutral buoyancy technique) or by using supporting disks with very small radius (micro- or millimetric liquid bridges).

The experiments described in this article were performed using an experimental facility in which the three previously mentioned effects that contribute to the magnitude of the Bond number can be, within limits, independently controlled. In this facility, the neutral buoyancy technique is employed and $\Delta \rho$ is controlled by selecting the appropriate density of the surrounding fluid. Support disks of different diameters can be also used and the liquid column is mounted on a centrifuge so that the magnitude of the acceleration acting on the bridge can be adjusted by varying the rotation rate. The accelerations that must be accounted for are gravity plus the centripetal acceleration due to the solid-body rotation of the liquid bridge.

It must be pointed out that the Bond number is not constant along the liquid column. This is because the liquid bridge rotates as a solid body and the centripetal acceleration varies with the distance to the rotation axis. However, for certain conditions the effect of the Bond number gradient can be negligible as shown in the Appendix.

The range of validity of Eq. (4) was tested using a numerical approach and by laboratory experiments. Our numerical approach employed the code SURFACE EVOLVER and the results are presented in Sec. II. The experimental apparatus and results are described in Sec. III. The results are discussed in Sec. IV.

\section{NUMERICAL APPROACH}

The range of validity of Eq. (4) was investigated by calculating the stability of equilibrium configurations using a numerical procedure that involved solving for energyminimizing surface configurations. We considered liquid bridges with constant surface tension and held between rigid coaxial disks of radius $R$ that are separated by a distance $L$ and subjected to steady nonaxial acceleration represented by axial and lateral Bond numbers (see Fig. 1). The stability limits of these bridges were obtained using the following procedure. It is assumed that the surface of the bridge is anchored to the edges of two coaxial circular disks and that the bridge volume is constant. Both lateral acceleration (i.e., gravity directed perpendicular to the bridge axis) and axial acceleration were considered. We first considered a liquid bridge of cylindrical volume, $v=V-1=0$. For this fixed volume, the objective was to find stable configurations of the bridges for both axial and lateral accelerations. To determine the location of the stability boundary for given axial and lateral Bond numbers, $B_{a}$ and $B_{l}$, we sought the maximum stable slenderness, $\Lambda_{\max }$. For bridges above the stability limit defined by $\Lambda_{\max }$, the bridges break and no stable bridge shape is found. Below the stability boundary, the bridges maintain their integrity and reach a minimum energy configuration. Using a simple iterative search technique we were able to find the maximum stable slenderness corresponding to the stability boundary. For fixed $B_{a}$ and $B_{l}$ we selected a value of slenderness, $\Lambda$, and computed the minimum energy configuration. If the bridge reached a minimum energy without breaking we then increased the value of $\Lambda$ and repeated the calculations. This procedure continued until the bridge broke.

The problem was approached using the code SURFACE EVOLVER. ${ }^{11}$ SURFACE EVOLVER seeks the shape of an energyminimizing surface subject to given boundary conditions and constraints. The surface is locally discretized using triangular elements. The vertex coordinates $\boldsymbol{X}$ of the elements are points in 3D Euclidean space and are used to parametrize the surface. SURFACE EVOLVER minimizes the energy $E(\boldsymbol{X})$ associated with each surface element. Through evaluation of the energy gradient at a given $\boldsymbol{X}$, SURFACE EVOLVER seeks the minimum by proceeding down the direction of the steep- 
TABLE I. Reduced $b_{a}$ and $b_{l}$ for cylindrical liquid bridges $(V=1)$. Boldface rows were calculated with an axial Bond number gradient corresponding to rotation.

\begin{tabular}{lllll}
\hline \hline \multicolumn{1}{c}{$B_{a}$} & $B_{l}$ & $\Lambda_{\max }$ & $b_{a}$ & $b_{l}$ \\
\hline 0 & 0.20 & 2.85 & 0 & 1.03 \\
0.001 & 0.26 & 2.57 & 0.029 & 0.957 \\
0.0025 & 0.22 & 2.68 & 0.1 & 0.9 \\
0.003 & 0.2 & 2.73 & 0.14 & 0.868 \\
$\mathbf{0 . 0 0 3}$ & $\mathbf{0 . 2}$ & $\mathbf{2 . 7 3 5}$ & $\mathbf{0 . 1 4 5}$ & $\mathbf{0 . 8 7 3}$ \\
0.003 & 0.18 & 2.78 & 0.173 & 0.833 \\
0.004 & 0.17 & 2.785 & 0.235 & 0.793 \\
0.005 & 0.155 & 2.805 & 0.32 & 0.74 \\
0.005 & 0.14 & 2.83 & 0.36 & 0.7 \\
0.006 & 0.13 & 2.835 & 0.443 & 0.654 \\
$\mathbf{0 . 0 0 6}$ & $\mathbf{0 . 1 3}$ & $\mathbf{2 . 8 3 5}$ & $\mathbf{0 . 4 4 3}$ & $\mathbf{0 . 6 5 4}$ \\
0.006 & 0.12 & 2.855 & 0.49 & 0.624 \\
0.007 & 0.11 & 2.853 & 0.565 & 0.57 \\
0.008 & 0.1 & 2.848 & 0.63 & 0.514 \\
0.008 & 0.09 & 2.863 & 0.68 & 0.475 \\
0.009 & 0.08 & 2.856 & 0.74 & 0.417 \\
0.010 & 0.07 & 2.854 & 0.81 & 0.36 \\
0.010 & 0.05 & 2.869 & 0.88 & 0.267 \\
0.012 & 0.04 & 2.845 & 0.93 & 0.204 \\
$\mathbf{0 . 0 1 2}$ & $\mathbf{0 . 0 4}$ & $\mathbf{2 . 8 4 5}$ & $\mathbf{0 . 9 3}$ & $\mathbf{0 . 2 0 4}$ \\
0.012 & 0.02 & 2.858 & 0.995 & 0.105 \\
\hline \hline
\end{tabular}

est slope using either a steepest descent or a conjugate gradient method. It is often advantageous to change the type of descent method during the iterative process. At each iteration, the force on each element vertex is calculated from the local energy gradient evaluated at that vertex. This force yields the direction of motion of the element and must also account for global constraints due to boundaries or volume preservation. The actual motion is found by multiplication of the resultant force by an optimum scale factor that is calculated at each step and each element is then moved to its new location. This procedure is repeated until the total energy is minimized. We tested the ability of SURFACE EVOLVER to find the stability limits for axisymmetric bridges subject to axial acceleration. In general, it was necessary to refine the triangular mesh frequently. Each minimization generally took several hundred iterations. Energy changes of one part in $10^{8}$ were assumed sufficient to ensure that equilibrium had been reached (see comments in Ref. 11).

The computational results for cylindrical volume bridges are listed in Table I. Here the axial Bond number $B_{a}$, the lateral Bond number $B_{l}$, and the maximum slenderness $\Lambda_{\max }$ for which the liquid bridge is stable, are shown. The reduced axial and lateral Bond numbers, $b_{a}$ and $b_{l}$, calculated with Eq. (2) and (3) are also shown in Table I. For certain cases (indicated in the tables) calculations were carried out for situations with an axial gradient in the Bond number corresponding to the centrifuge-type rotation used in the experiments described in the next section and in the Appendix.

Points that represent the limits of stable liquid bridges, computed with SURFACE EVOLVER, are plotted in Fig. 2(a), together with the predictions of Eq. (4). In this case, the agreement between numerical and theoretical results is very good. A sequence of the stable shapes calculated for the maximum stable slenderness is shown in Fig. 2(b).
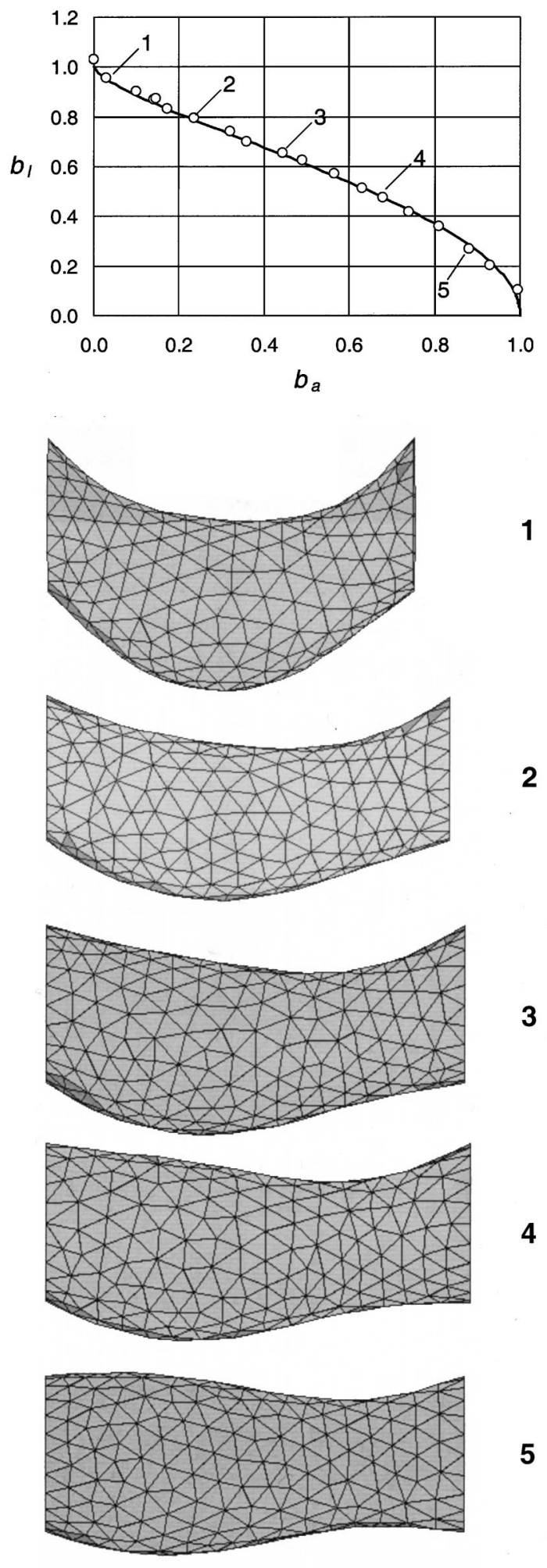

3

4

FIG. 2. (a) Stability limits for a cylindrical volume bridge subject to steady nonaxisymmetric acceleration in terms of the dimensionless reduced axial and lateral Bond numbers, $b_{a}$ and $b_{l}$. Symbols denote the calculated results obtained using SURFACE EVOLVER (Ref. 11) and correspond to the values listed in Table I. The solid line was obtained using Eq. (4). Points marked by numbers refer to the calculated shapes shown in Fig. 2(b). (b) Sequence of stable shapes close to the stability limit calculated for the maximum stable slenderness using SURFACE EVOLVER. The numbers correspond to the numbered points in $\left(b_{a}, b_{l}\right)$-space shown in Fig. 2(a). 
TABLE II. Reduced $b_{a}$ and $b_{l}$ for $V=1.1$ liquid bridges. Boldface rows were calculated with an axial Bond number gradient corresponding to rotation.

\begin{tabular}{lllll}
\hline \hline$B_{a}$ & $B_{l}$ & $\Lambda_{\max }$ & $b_{a}$ & $b_{l}$ \\
\hline 0.004 & 0.3 & 2.40 & 0.063 & 0.88 \\
$\mathbf{0 . 0 0 4}$ & $\mathbf{0 . 3}$ & $\mathbf{2 . 4 0}{ }^{*}$ & $\mathbf{0 . 0 6 3}^{*}$ & $\mathbf{0 . 8 8}^{*}$ \\
0.01 & 0.27 & 2.47 & 0.178 & 0.826 \\
0.015 & 0.23 & 2.54 & 0.306 & 0.735 \\
0.021 & 0.18 & 2.61 & 0.495 & 0.604 \\
$\mathbf{0 . 0 2 1}$ & $\mathbf{0 . 1 8}$ & $\mathbf{2 . 6 0}$ & $\mathbf{0 . 4 8 6}$ & $\mathbf{0 . 6 0}$ \\
0.026 & 0.14 & 2.64 & 0.655 & 0.48 \\
0.032 & 0.1 & 2.64 & 0.806 & 0.343 \\
0.036 & 0.07 & 2.64 & 0.91 & 0.24 \\
0.04 & 0.03 & 2.63 & 0.985 & 0.102 \\
$\mathbf{0 . 0 4}$ & $\mathbf{0 . 0 3}$ & $\mathbf{2 . 6 2 5}$ & $\mathbf{0 . 9 8}$ & $\mathbf{0 . 1 0}$ \\
\hline \hline
\end{tabular}

To test the extent to which (4) reliably predicts the stability of bridges with $v \neq 0$, we repeated our calculations with liquid bridges slightly different from the reference configuration $\left(\Lambda \sim \pi, v \sim 0, B_{a} \sim 0, B_{l} \sim 0\right)$. The results for volumes $V=1.1, V=1.2$, and $V=1.3$ are listed in Tables II-IV. These results are plotted in Fig. 3 together with the theoretical results. For liquid bridges with relative volumes as large as $V=1.2$ the agreement between numerical and theoretical results is good. For larger volumes, the ability of Eq. (4) to predict the stability limit deteriorates. For a limited number of calculations carried out for volumes less than 1 , the reliability of Eq. (4) deteriorated for $V<0.9$.

\section{APPARATUS AND EXPERIMENTAL RESULTS}

To perform the experiments described in the following, an experimental facility, as sketched in Fig. 4, has been used. The apparatus consists of a liquid bridge cell mounted on a horizontal, rotating platform. The platform can rotate at any prescribed angular velocity within the range $0-1.05 \mathrm{rad}_{\mathrm{s}}{ }^{-1}$ $(0-10 \mathrm{rpm})$ with an accuracy of $\pm 2 \times 10^{-3} \mathrm{rad}_{\mathrm{s}}{ }^{-1}( \pm 0.02$ $\mathrm{rpm})$. The rotating platform is a metallic beam $1.5 \mathrm{~m}$ in radius, mounted on a support structure where the control electronics and the electric motor used to rotate the beam are located.

Experiments were performed using the so-called neutral buoyancy or plateau technique. Here the liquid column is formed inside another immiscible liquid (a surrounding bath). For neutral buoyancy the bath liquid has practically the same density as the bridge liquid. The bath density (and, thus, the Bond number) can be changed by adjusting the composition of the bath liquid.

TABLE III. Reduced $b_{a}$ and $b_{l}$ for $V=1.2$ liquid bridges.

\begin{tabular}{lllll}
\hline \hline \multicolumn{1}{c}{$B_{a}$} & $B_{l}$ & $\Lambda_{\max }$ & $b_{a}$ & $b_{l}$ \\
\hline 0.008 & 0.35 & 2.20 & 0.082 & 0.87 \\
0.02 & 0.3 & 2.33 & 0.24 & 0.787 \\
0.03 & 0.25 & 2.41 & 0.404 & 0.68 \\
0.04 & 0.19 & 2.48 & 0.598 & 0.535 \\
0.05 & 0.15 & 2.49 & 0.759 & 0.425 \\
0.06 & 0.11 & 2.49 & 0.91 & 0.28 \\
\hline \hline
\end{tabular}

TABLE IV. Reduced $b_{a}$ and $b_{l}$ for $V=1.3$ liquid bridges. Boldface rows were calculated with an axial Bond number gradient corresponding to rotation.

\begin{tabular}{lllll}
\hline \hline \multicolumn{1}{c}{$B_{a}$} & $B_{l}$ & $\Lambda_{\max }$ & $b_{a}$ & $b_{l}$ \\
\hline 0.025 & 0.45 & 1.85 & 0.164 & 0.944 \\
$\mathbf{0 . 0 2 5}$ & $\mathbf{0 . 4 5}$ & $\mathbf{1 . 8 5}$ & $\mathbf{0 . 1 6 4}$ & $\mathbf{0 . 9 4 4}$ \\
0.035 & 0.4 & 1.9 & 0.25 & 0.864 \\
0.04 & 0.35 & 2.12 & 0.336 & 0.798 \\
0.045 & 0.3 & 2.24 & 0.429 & 0.713 \\
0.05 & 0.26 & 2.31 & 0.516 & 0.634 \\
0.055 & 0.24 & 2.33 & 0.581 & 0.59 \\
0.06 & 0.22 & 2.35 & 0.65 & 0.54 \\
0.065 & 0.18 & 2.38 & 0.729 & 0.451 \\
0.07 & 0.12 & 2.44 & 0.846 & 0.31 \\
0.08 & 0.1 & 2.41 & 0.93 & 0.25 \\
$\mathbf{0 . 0 8}$ & $\mathbf{0 . 1}$ & $\mathbf{2 . 4 0}$ & $\mathbf{0 . 9 3}$ & $\mathbf{0 . 2 5 3}$ \\
\hline \hline
\end{tabular}

The Liquid Bridge Cell (LBC), where the liquid bridge is formed, is a tight chamber connected to a calibrated syringe. The LBC test chamber is a $0.04 \mathrm{~m} \times 0.04 \mathrm{~m} \times 0.04 \mathrm{~m}$ aluminum cube, with two opposite faces made of a transparent, plastic material. This allows visualization of the liquid bridge. The liquid bridge is formed between two equal disks $0.01 \mathrm{~m}$ in diameter. One of the disks is connected to the piston of the syringe and can be displaced along its axis by using a micrometer screw. The remaining disk is fixed to the opposite side of the test chamber such that both disks remain in coaxial alignment whatever their separation distance. Liquid is injected and removed from the liquid bridge through a hole in the center of the moving disk which connects it with the syringe. The diameter of the syringe is equal to the diameter of the disks and the moving disk is mounted at one end of the piston syringe. Thus, the amount of liquid injected or removed when the disk is displaced causes the volume of the liquid column to be cylindrical within $0.1 \%$ accuracy $[V=(1 \pm 0.001) 2 \pi \Lambda]$ regardless of the distance separating the disks.

Two quick-disconnect valves are used to fill the test chamber with the surrounding liquid. LBC as well as the

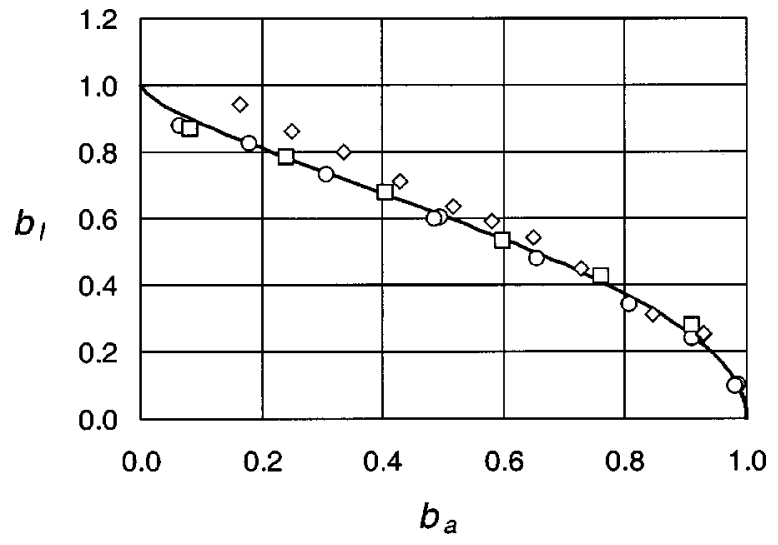

FIG. 3. Stability limits for noncylindrical volume bridges subject to steady nonaxisymmetric acceleration in terms of the dimensionless reduced axial and lateral Bond numbers, $b_{a}$ and $b_{l}$. Symbols denote the calculated results using SURFACE EVOLVER, according to the following key: $V=1.1$ (circle), $V=1.2$ (rectangle), $V=1.3$ (rhomb), and the solid line is Eq. (4). 


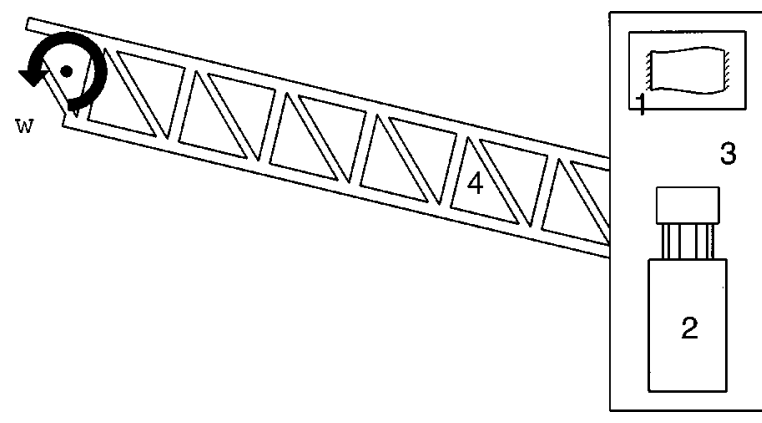

FIG. 4. Top view of the experimental arrangement: 1. liquid bridge cell (LBC), 2. CCD camera, 3. supporting plate, 4. rotating platform.

illumination system and a Charge Coupled Device (CCD) video camera are mounted on a plate, which in turn is mounted on the rotating platform. The liquid bridge axis is horizontal and perpendicular to the axis of rotation. The CCD camera is connected to a small television (TV) transmitter which sends signals to a TV receiver placed a few meters away at the control station.

In each experiment, the experimental procedure went as follows: first, the moving disk is axially displaced until it becomes very close to the lower one. Then the test chamber of the liquid bridge cell is filled with the surrounding bath of the desired density. A small liquid bridge that fills the gap between both disks is formed. There are four liquid bridge cells, and the above operation is repeated for each one. The $\mathrm{LBC}$ is mounted on the plate, with $Z^{*}$ being the distance between the rotation axis and the center of the LBC. The moving disk is displaced axially until the prescribed slenderness is reached. Working liquid is simultaneously injected into the liquid bridge to keep its volume cylindrical (i.e., $v$
TABLE V. Variation with the difference in densities between the surrounding bath and the working liquid, $\Delta \rho=\rho_{\mathrm{sb}}-\rho_{\mathrm{wl}}$, of the interfacial tension between both liquids, $\sigma$.

\begin{tabular}{cc}
\hline \hline$\Delta \rho \pm 0.2\left(\mathrm{~kg} / \mathrm{m}^{3}\right)$ & $\sigma \pm 0.002(\mathrm{~N} / \mathrm{m})$ \\
\hline 2.0 & 0.011 \\
8.2 & 0.015 \\
14.5 & 0.019 \\
19.2 & 0.021 \\
26.4 & 0.025 \\
29.8 & 0.028 \\
30.8 & 0.029 \\
\hline
\end{tabular}

$=0)$ as the distance between the disks increases. Once the CCD camera and the TV transmitter are switched on, the rotating platform is rotated at a very low rate and the rotation velocity is slowly increased until the liquid column breaks. The rotating platform is then stopped. The LBC is replaced, a new liquid bridge with the desired slenderness is formed, and the entire process is repeated.

Note that, when the platform is set into rotation at an angular velocity $\omega$, the accelerations acting on the liquid column include gravity as well as the centrifugal acceleration. Thus, the axial and lateral Bond numbers are: $B_{a}$ $=\Delta \rho \omega^{2} Z^{*} R^{2} / \sigma$ and $B_{l}=\Delta \rho g R^{2} / \sigma$, respectively. It must be pointed out that formally, the axial Bond number is not constant along the liquid bridge. It varies linearly with the distance to the axis of rotation. However, for some practical situations, this linear effect is negligible, as demonstrated in the Appendix.

All experiments were performed at a temperature of 25 $\pm 1{ }^{\circ} \mathrm{C}$. The density of the working liquid (dimethyl-silicone oil POLISIL M50), $\rho_{\mathrm{wl}}$, at this temperature is

TABLE VI. Experimental results for maximum stable slenderness, $\Lambda$ and $V=1$. The values of the axial and lateral Bond numbers, $B_{a}$ and $B_{l}$, respectively, are the values at which the maximum slenderness is reached. Also given are the reduced axial and lateral Bond numbers, $b_{a}$ and $b_{l}$. In this table $b_{1 \mathrm{~T}}$ is the calculated reduced lateral Bond number based on the measured $b_{a}: b_{\mathrm{IT}}=\left(1-\left(b_{a}\right)^{2 / 3}\right)^{1 / 2}$.

\begin{tabular}{ccccccccccccc}
\hline \hline$\Lambda$ & $B_{a}$ & $B_{l}$ & $b_{a}$ & $b_{l}$ & $b_{\mathrm{IT}}$ & $\Lambda$ & $B_{a}$ & $B_{l}$ & $b_{a}$ & $b_{l}$ & $b_{\mathrm{IT}}$ \\
\hline 1.1 & 0.217 & 0.125 & 0.932 & 0.238 & 0.214 & 2.3 & 0.013 & 0.261 & 0.215 & 0.791 \\
1.2 & 0.177 & 0.177 & 0.820 & 0.354 & 0.352 & 2.3 & 0.014 & 0.260 & 0.225 & 0.790 & 0.794 \\
1.4 & 0.165 & 0.095 & 0.899 & 0.200 & 0.261 & 2.3 & 0.014 & 0.261 & 0.230 & 0.791 & 0.790 \\
1.6 & 0.135 & 0.135 & 0.135 & 0.884 & 0.303 & 2.3 & 0.015 & 0.260 & 0.239 & 0.790 & 0.784 \\
2.0 & 0.034 & 0.261 & 0.353 & 0.679 & 0.708 & 2.3 & 0.015 & 0.261 & 0.247 & 0.791 & 0.778 \\
2.0 & 0.035 & 0.260 & 0.359 & 0.678 & 0.704 & 2.4 & 0.007 & 0.260 & 0.145 & 0.841 & 0.851 \\
2.0 & 0.044 & 0.247 & 0.452 & 0.644 & 0.641 & 2.4 & 0.007 & 0.261 & 0.145 & 0.843 & 0.851 \\
2.1 & 0.026 & 0.260 & 0.303 & 0.710 & 0.741 & 2.4 & 0.008 & 0.251 & 0.157 & 0.811 & 0.842 \\
2.1 & 0.026 & 0.261 & 0.309 & 0.711 & 0.737 & 2.4 & 0.008 & 0.260 & 0.155 & 0.841 & 0.844 \\
2.1 & 0.028 & 0.261 & 0.334 & 0.711 & 0.720 & 2.4 & 0.008 & 0.261 & 0.150 & 0.843 & 0.847 \\
2.1 & 0.030 & 0.260 & 0.356 & 0.710 & 0.705 & 2.4 & 0.009 & 0.261 & 0.158 & 0.843 & 0.841 \\
2.1 & 0.033 & 0.251 & 0.389 & 0.685 & 0.684 & 2.4 & 0.010 & 0.251 & 0.196 & 0.811 & 0.814 \\
2.2 & 0.017 & 0.260 & 0.240 & 0.747 & 0.784 & 2.4 & 0.016 & 0.218 & 0.314 & 0.705 & 0.734 \\
2.2 & 0.020 & 0.261 & 0.279 & 0.748 & 0.757 & 2.4 & 0.017 & 0.218 & 0.334 & 0.705 & 0.720 \\
2.2 & 0.021 & 0.260 & 0.284 & 0.747 & 0.754 & 2.4 & 0.044 & 0.006 & 0.883 & 0.019 & 0.282 \\
2.2 & 0.022 & 0.260 & 0.304 & 0.747 & 0.740 & 2.5 & 0.003 & 0.260 & 0.082 & 0.904 & 0.900 \\
2.2 & 0.023 & 0.261 & 0.315 & 0.748 & 0.733 & 2.5 & 0.003 & 0.261 & 0.079 & 0.906 & 0.903 \\
2.2 & 0.024 & 0.251 & 0.329 & 0.720 & 0.723 & 2.5 & 0.004 & 0.260 & 0.087 & 0.904 & 0.896 \\
2.2 & 0.025 & 0.251 & 0.343 & 0.720 & 0.714 & 2.5 & 0.004 & 0.261 & 0.093 & 0.906 & 0.892 \\
2.3 & 0.017 & 0.251 & 0.276 & 0.762 & 0.759 & 2.5 & 0.005 & 0.251 & 0.122 & 0.872 & 0.868 \\
2.3 & 0.024 & 0.218 & 0.389 & 0.662 & 0.683 & 2.5 & 0.010 & 0.218 & 0.244 & 0.758 & 0.781 \\
2.3 & 0.033 & 0.188 & 0.536 & 0.571 & 0.584 & 2.5 & 0.011 & 0.218 & 0.268 & 0.758 & 0.764 \\
2.3 & 0.009 & 0.261 & 0.142 & 0.791 & 0.853 & 2.5 & 0.039 & 0.022 & 0.951 & 0.076 & 0.182 \\
\hline \hline
\end{tabular}




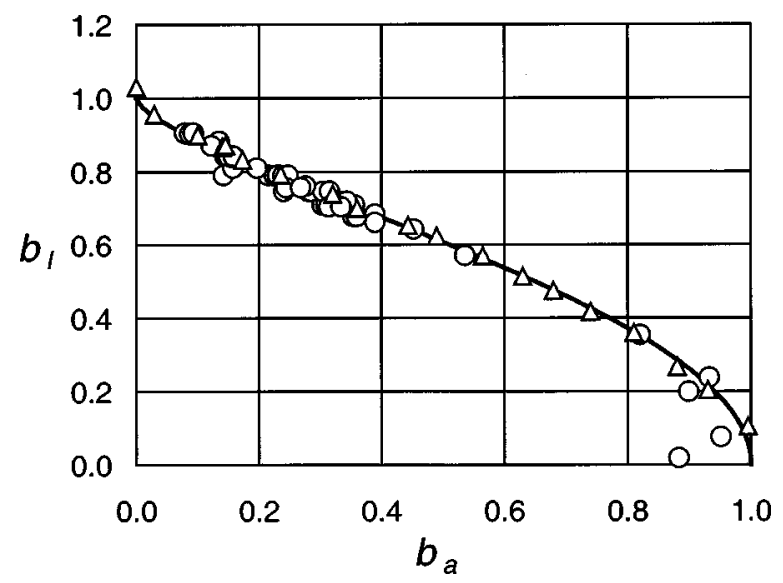

FIG. 5. Experimentally determined stability limits for a cylindrical volume bridge subject to steady nonaxisymmetric acceleration in terms of the dimensionless reduced axial and lateral Bond numbers, $b_{a}$ and $b_{l}$. Open circles denote experimental results, open triangles denote numerical results with $V=1$, and the solid line is Eq. (4)

$\rho_{\mathrm{wl}}=959.4 \pm 0.1 \mathrm{~kg} \cdot \mathrm{m}^{-3}$. For the surrounding bath seven different mixtures of methanol and distilled water were used. The densities of the surrounding liquid, $\rho_{\mathrm{sb}}$, used in the experiments were $\rho_{\mathrm{sb}}=961.4,967.6,973.9,978.6,985.8$, 989.2, and $990.2 \pm 0.1 \mathrm{~kg} \cdot \mathrm{m}^{-3}$ (note that, since the LBC test chamber is tight, no alcohol evaporation occurs and the surrounding bath density is constant for the duration of the experiment).

To calculate the value of the interfacial tension between the bridge liquid and the surrounding bath, the same procedure explained in Meseguer et al. ${ }^{10}$ was used. For each value of the surrounding bath density, different slenderness liquid bridges with vertical axes were formed. The deformation of the liquid bridge interface depends on the value of the Bond number. Thus, by fitting a second-order approximation of the liquid bridge interface to the experimental liquid bridge contours, the value of the Bond number, $B_{a}$, is obtained. The value of the interfacial tension is then obtained indirectly: $\sigma=\Delta \rho g R^{2} / B_{a}$, where $\Delta \rho=\rho_{\mathrm{sb}}-\rho_{\mathrm{wl}}$ and $g$ is the gravity acceleration $\left(g=9.81 \mathrm{~m} . \mathrm{s}^{-2}\right)$. The results obtained, $\sigma$ vs. $\Delta \rho$, are shown in Table $\mathrm{V}$. The value of the interfacial tension varies as the surrounding bath density varies, because the ratio of alcohol to water of the mixture changes. To test whether sedimentation of the surrounding bath occurs during centrifugation, interfacial tension measurements were made after centrifugation of a stable liquid bridge for a few cases. The tests were carried out under the same conditions as the stability tests. The measured values of interfacial tension were found to be the same.

Experimental results obtained by using different distances to the rotation axis ( $Z^{*}$ varies from 0.3 to $1.2 \mathrm{~m}$ ) are listed in Table VI. The maximum slenderness, $\Lambda_{\max }$, the lateral Bond number, $B_{l}=\Delta \rho g R^{2} / \sigma$, and the axial Bond number, $B_{a}=\Delta \rho \omega^{2} Z^{*} R^{2} / \sigma$, are given. (Here $\omega$ is the maximum rotation velocity for which the liquid bridge is stable.) The reduced axial and lateral Bond numbers, $b_{a}$ and $b_{l}$, given by Eqs. (2) and (3) are also shown in Table VI.

The variation with the reduced axial Bond number of the reduced lateral Bond number that results from expression (4), as well as experimental and numerical results, has been represented in Fig. 5. As it can be observed, at least in the case of cylindrical liquid bridges, the agreement between experimental and theoretical results is good. There is, however, an experimental point $\left(b_{a}=0.883, b_{l}=0.019\right)$ which lies far from the theoretical prediction. The reason for this discrepancy could be that this point was obtained at a higher rotation speed. At this very high rotation velocity some spurious vibration of the rotating arm was observed, and it is probable that this vibration caused breakage of the liquid column before the static stability limit was reached.

\section{CONCLUSIONS}

An experimental and computational study of the stability of liquid bridges between equal disks has been performed. The study focused on determining the range of validity of a self-similar solution for the stability limits of slender liquid bridges subject to steady nonaxisymmetric acceleration. In addition to the results described in Sec. II, we carried out computations using the same values of $B_{a}$ and $B_{l}$ as the experimental ones and obtained the maximum stable slenderness. The solution appears to a reasonable approximation for dimensionless volumes $V=V^{*} /\left(\pi R^{2} L\right)$ that are close to a cylindrical volume $(V=1)$.

\section{ACKNOWLEDGMENTS}

This work was supported by the Spanish Comision Interministerial de Ciencia y Tecnología (CICYT Project No. ESP 95-0029), and by the National Aeronautics and Space Administration through grants NAG3-1864 and NAG3-2160.

\section{APPENDIX: EFFECT OF BOND NUMBER GRADIENT ON $\Lambda_{\max }$}

Let us assume a liquid bridge of cylindrical volume, $V^{*}=\pi R^{2} L$, placed between two equal, coaxial, parallel disks, $R$ being the radius of the disks and $L$ the distance between them. The liquid bridge, as sketched in Fig. 6, is rotating as a solid body with angular velocity $\omega$. The rotation axis is parallel to the local gravity vector. Both axes the liquid bridge and the rotation axis are perpendicular and have a common point $O$. Let $Z^{*}$ be the distance between the axis of rotation and the center of the liquid column, where the local coordinate axes $\left(z^{*}, r^{*}, \theta\right)$ are located.

The equation defining the shape of the interface of the liquid bridge, $F^{*}=F^{*}\left(z^{*}, \theta\right)$, must express the balance between capillary and hydrostatic forces:

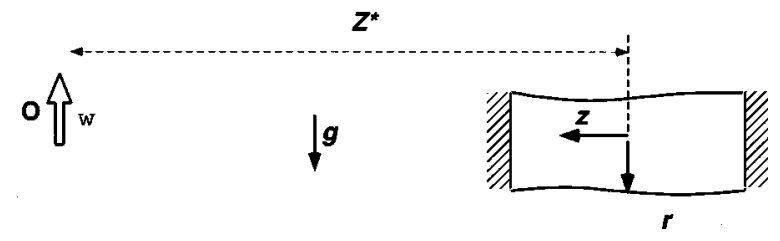

FIG. 6. Sketch of the liquid bridge as a rotating body showing the distance $Z^{*}$ between the rotation axis and the center of the liquid column. 


$$
\begin{aligned}
& \sigma\left(\zeta_{1}^{-1}+\zeta_{1}^{-1}\right)+P^{*}+\Delta \rho\left[g F^{*} \cos \theta+\omega^{2}\left(\frac{1}{2} z^{* 2}-Z^{*} z^{*}\right)\right. \\
& \left.\quad+\frac{1}{2} \omega^{2} F^{* 2} \sin ^{2} \theta\right]=0 .
\end{aligned}
$$

Here $\left(\zeta_{1}^{-1}+\zeta_{2}^{-1}\right)$ is the curvature of the interface, $\sigma$ is the interfacial tension, $P^{*}$ is a constant that fixes the origin for the pressure, and $\Delta \rho$ is the density difference between the working liquid and the surrounding fluid.

We now introduce the dimensionless variables $(z$ $=z^{*} / R, \quad \Lambda=L /(2 R), \quad V=V^{*} /\left(\pi R^{2} L\right), \quad F=F^{*} / R, \quad P$ $\left.=P^{*} R / \sigma, \ldots\right)$ and define the different "Bond numbers" appearing in the formulation as follows:

axial Bond number: $B_{a}=\Delta \rho R^{2} \omega^{2} Z^{*} / \sigma$,

lateral Bond number: $B_{l}=\Delta \rho R^{2} g / \sigma$,

axial gradient of Bond number: $D_{a}=\Delta \rho R^{3} \omega^{2} /(2 \sigma)$.

The nondimensional differential equation defining the equilibrium interface shapes now reads:

$$
M(F)+P-B_{a} z+B_{l} F \cos \theta+D_{a}\left(z^{2}+F^{2} \sin ^{2} \theta\right)=0,
$$

where

$$
\begin{aligned}
M(F)=\{ & F\left[1+\left(F_{z}\right)^{2}\right]\left[F_{\theta \theta}-F\right]+F F_{z z}\left[F^{2}+\left(F_{\theta}\right)^{2}\right] \\
& \left.-2 F_{\theta}\left[F_{\theta}+F F_{z} F_{z \theta}\right]\right\} \cdot\left\{F^{2}\left[1+\left(F_{z}\right)^{2}\right]\right. \\
& \left.+\left(F_{\theta}\right)^{2}\right\}^{-3 / 2},
\end{aligned}
$$

and the boundary conditions are:

$$
\begin{aligned}
& F( \pm \Lambda, \theta)=1, \quad F(z, \theta+2 \pi)=F(z, \theta), \\
& \frac{1}{2} \int_{-\Lambda}^{\Lambda}\left(\int_{0}^{2 \pi} F^{2} d \theta\right) d z=2 \pi \Lambda .
\end{aligned}
$$

It can be observed that if the term in $D_{a}$ is neglected in Eq. (A3), the formulation is similar to that of a liquid bridge of cylindrical volume subjected to a constant acceleration with both axial and lateral components. This last problem was analyzed in Ref. 4 using standard bifurcation theory and the following asymptotic expression for the maximum stable slenderness, $\Lambda_{\max }$, was obtained:

$$
\Lambda_{\max }=\pi\left[1-\left(\frac{3}{2}\right)^{4 / 3} B_{a}^{2 / 3}+\frac{1}{2} v-\frac{\pi^{2}}{4} B_{l}^{2}\right] .
$$

To properly account for the nonuniformity of the acceleration a similar asymptotic expression for a liquid bridge with an axial Bond number gradient has been calculated. (Additional details can be obtained upon request.) In this case, the expression giving the maximum slenderness of the liquid column is now:

$$
\Lambda_{\max }=\pi\left[1-\left(\frac{3}{2}\right)^{4 / 3}\left(B_{a}+\phi_{B D} B_{a} D_{a}\right)^{2 / 3}+\frac{1}{2} v-\frac{\pi^{2}}{4} B_{l}^{2}\right]
$$

with $\phi_{B D}=50 / 4-4 \pi^{2} / 3$.

Note that, according to the above expression, the Bond number gradient is a second-order effect which becomes negligible in most practical situations. The second-order term $\phi_{B D} B_{a} D_{a}$ is approximately $D_{a}$ times the value of axial Bond number. On the other hand, from the definition of the axial Bond number, $B_{a}$, Equation (A2a), and the definition of the axial Bond number gradient, $D_{a}$, expression (A2c), one gets $D_{a}=\left(R / Z^{*}\right) B_{a}$.

In the experiments reported here (see Sec. III), the maximum value of $B_{a}$ is approximately $2 \times 10^{-2}$. The supporting disks were $5 \mathrm{~mm}$ in radius and the minimum distance between the liquid bridge and the rotation axis was $Z^{*}$ $=300 \mathrm{~mm}$. Therefore, the maximum value of the axial acceleration gradient effect under consideration was $D_{a}$ $=\left(R / Z^{*}\right) B_{a} \approx 3 \times 10^{-4}$. This means that the axial acceleration gradient effect is $10^{-4}$ times less important than the effect of the axial Bond number. Consequently, Eq. (A4) rather than Eq. (A5) can be used to fit the experimental results.

${ }^{1}$ J. Meseguer, L. A. Slobozhanin, and J. M. Perales, "A review on the stability of liquid bridges," Adv. Space Res. 16, 5 (1994).

${ }^{2}$ S. R. Coriell, S. C. Hardy, and M. R. Cordes, "Stability of liquid zones," J. Colloid Interface Sci. 60, 126 (1977).

${ }^{3}$ J. M. Perales, "Non-axisymmetric effects on long liquid bridges," Acta Astron. 15, 561 (1987).

${ }^{4}$ J. Meseguer, N. A. Bezdenejnykh, J. M. Perales, and P. Rodríguez de Francisco, "Theoretical and experimental analysis of stability limits of non-axisymmetric liquid bridges under microgravity conditions,' Microgravity Sci. Technol. 8, 2 (1995)

${ }^{5}$ H. Chen and M. Z. Saghir, "Nonaxisymmetric equilibrium shapes of the liquid bridge," Microgravity Sci. Technol. 7, 12 (1994).

${ }^{6}$ A. Laverón-Simavilla and J. M. Perales, "Equilibrium shapes of nonaxisymmetric liquid bridges of arbitrary volume in gravitational fields and their potential energy,"' Phys. Fluids 7, 1204 (1995).

${ }^{7}$ A. Laverón-Simavilla and E. Checa, "Effect of a lateral gravitational field on the non-axisymmetric equilibrium shapes of liquid bridges held between eccentric disks and of volumes equal to those of cylinders," Phys. Fluids 9, 817 (1997)

${ }^{8}$ J. I. D. Alexander, S. Delafontaine, A. Resnick, and W. C. Carter, "Stability of non-axisymmetric liquid bridges," Microgravity Sci. Technol. 9, 193 (1996).

${ }^{9}$ J. I. D. Alexander, Y. Zhang, S. Delafontaine, and A. Fedoseyev, "Numerical Simulation of Liquid Bridge Dynamics and Statics," in Numerical Methods in Heat and Mass Transfer Problems (Institute for Problems in Mechanics, Russian Academy of Sciences, Moscow, 1997), pp.109-135.

${ }^{10}$ J. Meseguer, N. A. Bezdenejnykh, and P. Rodríguez de Francisco, "On the use of liquid bridges as accelerometers," Microgravity Sci. Technol. 9, 62 (1996).

${ }^{11}$ K. Brakke, “The surface evolver," Experimental Math. 1, 141 (1992). 